\title{
Cultural consensus modelling to understand the reproductive health needs of South African adolescent girls
}

\author{
J L Brown, ${ }^{1} \mathrm{PhD}$; J M Sales, ${ }^{2} \mathrm{PhD}$; C Sharp, ${ }^{3,4} \mathrm{PhD}$; J Cloete, ${ }^{4}$ MSocSci; M Lenka, ${ }^{4} \mathrm{BS} ; \mathrm{K}$ Rani, ${ }^{4} \mathrm{MA} ; \mathrm{P}$ Marime, ${ }^{4} \mathrm{BS}$ Hons; \\ I Ditlhare, ${ }^{4} \mathrm{BA}$; R Moqolo, ${ }^{4} \mathrm{BA}$; D Peterson, ${ }^{4} \mathrm{BA}$; L Marais, ${ }^{4} \mathrm{PhD}$ \\ ${ }^{1}$ Department of Psychiatry and Behavioral Neuroscience, University of Cincinnati College of Medicine, Cincinnati, Ohio, USA \\ ${ }^{2}$ Department of Behavioral Sciences and Health Education, Rollins School of Public Health, Emory University, Atlanta, Georgia, USA \\ ${ }^{3}$ Department of Psychology, University of Houston, Texas, USA \\ ${ }^{4}$ Centre for Development Support, Economic and Management Sciences, University of the Free State, Bloemfontein, South Africa
}

Corresponding author: J Brown (jennifer.brown2@uc.edu)

\begin{abstract}
Background. Cultural consensus modelling (CCM) is an approach whereby individuals define the boundaries regarding a set of knowledge or behaviours shared by a group within a culture using an ethnographic approach.

Objectives. To provide an overview of CCM methods and the application of CCM to examine South African adolescent girls' contraceptive and HIV prevention practices.

Methods. In phase 1 of a CCM approach, individuals respond to questions about their culture rather than their individual behaviours, allowing individuals to draw upon a shared cultural knowledge. Utilising these identified group beliefs, phase 2 asks individuals to rate the extent to which factors identified in phase 1 are valued. Phase 3 utilises qualitative interviews with key informants from phase 2 to gather in-depth information regarding the identified determinants of the health behaviour. Lastly, phase 4 of this approach conducts a quantitative survey to determine the extent to which cultural consensus model types are associated with differences in actual behaviours. Results. CCM data analytic approaches are described. Frequencies and descriptive statistics for the free listing are conducted. For phase 2, cultural consensus analysis is conducted to examine whether one or several consensus models exist and competence scores are calculated. Standard qualitative analysis approaches are utilised for phase 3. Phase 4 employs regression to examine the association between cultural models and an outcome of interest.

Conclusion. CCM provides a novel, culturally sensitive understanding of reproductive health practices among South African adolescent girls; CCM also has broad applicability to other adolescent health research domains.
\end{abstract}

S Afr J Child Health 2018;12(2 Suppl 1):S40-S43. DOI:10.7196/SAJCH.2018.v12i2.1500

Adolescence to young adulthood represents a developmental period when adolescent girls may initiate sexual decision-making. Preventing adolescent pregnancy is a key avenue for promoting the empowerment of women and girls as well as achieving the United Nations' Sustainable Development Goals. ${ }^{[1]}$ When adolescent girls have the right to decide when to marry and have children, they are more likely to lead healthier, more productive lives as adults. ${ }^{[2]}$ South African adolescent girls experience some of the highest rates worldwide of both unintended pregnancies and sexually transmitted infections (STIs), including the human immunodeficiency virus (HIV). ${ }^{[3-6]}$ One-third of South African adolescent girls will be pregnant by age 20, and South Africa accounts for one-third of all new HIV infections among young women worldwide. ${ }^{[3-6]}$ Unintended teenage pregnancy and/or HIV infection results in numerous adverse health and socio-economic consequences including elevated risk of maternal and infant mortality, decreased educational attainment, exacerbation of poverty, perpetuation of gendered power imbalances, and increased experiences of stigma and discrimination, among others. ${ }^{[7,8]}$ To reduce unintended pregnancies and STI/HIV and empower South African adolescent girls' sexual and reproductive health decision-making, interventions that consider relevant cultural factors are urgently needed for this population.

Reproductive health counselling to promote dual protection (use of one or more methods to prevent both pregnancy and STI/HIV) is included in South African national guidelines for contraceptive services. ${ }^{[9]}$ To date, there are limited empirical data characterising factors associated with the contraceptive practices of
South African adolescent girls, with a lack of studies examining dual protection. ${ }^{[6,10-13]}$ The few extant studies highlight the importance of condom attitudes, ${ }^{[10]}$ contraceptive knowledge, ${ }^{[6,14]}$ and challenges communicating with sexual partners, ${ }^{[11]}$ as factors influencing contraceptive decisions. While studies of individual-level factors facilitate understanding of personal decision-making processes, there is a paucity of research examining the broader cultural context that might influence South African adolescent girls' contraceptive and dual protection practices. ${ }^{[15]}$

Cultural consensus modelling (CCM) is a methodology to develop a culturally sensitive understanding of a given topic, with culture defined as learned and shared beliefs, behaviours or norms held by a group. ${ }^{[16]}$ CCM is an approach whereby individuals define the boundaries regarding a set of knowledge or behaviours (e.g. contraceptive use, dual protection use) shared by a group within a culture using an ethnographic approach. ${ }^{[16-18]}$ A particular strength of CCM is that it starts by allowing respondents to specify factors based on the experiences which they believe are important. Thus, CCM is locally appropriate, so enlisting the strengths of an ethnographic approach. However, ethnographic approaches can falter because they may leave unanswered questions about the extent to which identified factors are shared across individuals, the extent to which different patterns of shared factors cluster across groups, and how important those factors are for predicting outcomes of interest. The CCM approach thus captures many strengths of qualitative research methodologies as well as strengths of quantitative statistical approaches to collect rich mixed-methods data to identify, from the 
ground up, a set of culturally relevant factors for a given outcome. The aim of the present paper is to provide a description of CCM methods and corresponding data analytic approaches to highlight the potential utility of this methodology for examining other adolescent health domains. We illustrate the application of the CCM methodology from a study examining cultural factors associated with contraceptive and HIV prevention practices among Sesotho-speaking South African adolescent girls.

\section{Methods \\ Overview}

The CCM methodology is iterative in its approach, with results from initial study phases informing subsequent phases. CCM results in rich quantitative and qualitative data to characterise the cultural factors associated with a particular outcome. In the following, we describe the overall approach employed in each of the four CCM study phases: (i) phase 1: free listing; (ii) phase 2: rating survey; (iii) phase 3: in-depth qualitative individual interviews; and (iv) phase 4: quantitative survey. We illustrate the application of CCM methods for each study phase from a study examining factors associated with pregnancy and STI/HIV prevention practices among South African adolescent girls. We also describe the participants and procedures employed in this study.

\section{Participants}

In all study phases, South African adolescent girls were recruited from a community of adolescent girls in the urban areas of Mangaung Metropolitan Municipality in the Free State Province of South Africa. Sesotho-speaking adolescents were recruited as it is the mostly widely spoken language in this region $(51.9 \%)$, despite being a language spoken by a minority of South Africans nationwide $(7.6 \%) .^{[19]}$ Participants were recruited by Sesothospeaking research staff via community outreach and use of fliers posted in neighbourhoods where recruitment took place. Eligibility for all four study phases included being: (i) female; (ii) between the ages of 14 and 17; (iii) Sesotho-speaking; and (iv) resident in Mangaung. For phase 3, participants had to have also participated in phase 2. As participants were all under age 18, written parental consent and written youth assent were obtained from all participants. The region is an urban location with poor socio-economic characteristics and a high prevalence of teenage pregnancies and STI/HIV; there is limited research examining contraceptive and STI/HIV prevention practices in Free State Province relative to other South African locations. ${ }^{[19,20]}$ Thus, the study targeted a population in need of reproductive health intervention approaches to enhance dual protection use.

\section{Phase 1: Free listing \\ Overview of phase 1 approach}

In phase 1 of CCM, individuals respond to questions about their culture rather than their individual behaviours using free listing. Free listing is a simple technique that asks a respondent to list as many items as they can think of within a given domain; this allows participants to draw upon a shared cultural knowledge when responding to free listing prompts. ${ }^{[17]}$

\section{Participants and procedures}

A convenience sample of 50 participants (mean age 15.7 years) was recruited. The sample size was determined based on previous studies suggesting that 50 participants would result in data saturation using free listing. ${ }^{[17]}$ Participants answered sociodemographic questions utilising items derived from the South African census ${ }^{[19]}$ and responded to free listing prompts. Data were collected via a tablet computer with REDCap's mobile app. REDCap (Research Electronic Data Capture) is a secure, web-based application designed to support data capture for research studies; the REDCap mobile app allows offline data collection. ${ }^{[21]}$

\section{Free listing}

Participants responded to two free listing questions assessing methods used to: (i) prevent pregnancy; and (ii) prevent STI/HIV. Participants were asked, 'What do other adolescent girls in your community think are good ways to prevent [pregnancy; STI/HIV]? Please list all the different ways that other adolescent girls in your community think are good to prevent [pregnancy; STI/HIV].' Next, participants responded to two additional prompts examining factors that influence selection of pregnancy and STI/HIV prevention methods. These free listing questions were "What do other adolescent girls in your community look for in a method to prevent [pregnancy; STI/HIV]? Please list as many different factors as possible that other adolescent girls in your community look for in a method to prevent [pregnancy; STI/ HIV]? Thus, prompts were designed to generate a comprehensive list of specific pregnancy and STI/HIV prevention methods and factors influencing method selection that represent the broader cultural context of adolescent girls within this community.

\section{Phase 2: Rating phase}

\section{Overview of phase 2 approach}

Utilising the identified group beliefs from the phase 1 free listing, phase 2 of CCM asks individuals to rate the extent to which identified factors are valued by other similar individuals in their community. This process and corresponding data analytic approach identifies unique clusters of individuals (termed cultural consensus models) who place greater importance on particular factors. This method allows one to identify whether there is a single shared cultural model of factors influencing a particular outcome of interest. It also allows one to test whether there are multiple cultural models within a population. This phase produces a set of 'culturally correct' answers (i.e. clusters of similar answer patterns across items); these responses inform the questionnaire design and data analysis used in phase 4 .

\section{Participants and procedures}

Phase 2 asked a different convenience sample of participants $(N=100$; mean age 15.6) to rate the extent to which factors identified in phase 1 were valued in order to evaluate whether distinct cultural consensus models exist for different subpopulations. The sample size was determined on the basis of previous studies suggesting that 100 participants will result in an adequate sample size to conduct the cultural consensus analysis. ${ }^{[17]}$ Participants completed the rating questionnaire via the REDCap mobile app on a tablet computer.

\section{Rating questionnaire}

For each of the pregnancy (e.g. contraceptive pills, withdrawal) and STI/ HIV (e.g. STI/HIV testing, using male condoms) prevention methods identified in phase 1, participants rated how acceptable they believed each method was among other girls in their community. Participants rated the acceptability of each method from very unacceptable (1) to very acceptable (5). For each of the factors influencing pregnancy prevention strategy selection identified in the phase 1 free listing (e.g., avoiding weight gain, no dangerous side-effects), participants then rated how important each factor was to other girls in their community, from very unimportant (1) to very important (5).

\section{Phase 3: In-depth qualitative interviews}

\section{Overview of phase 3 approach}

Phase 3 of CCM uses qualitative interviews with key informant participants from phase 2 to gather in-depth information regarding the identified outcome of interest. Participants are asked about other similar members of their identified community, rather than providing their individual experiences. Respondents for phase 3 are selected 
because they either belong to different cultural consensus models (in the case of multiple cultural consensus models) or because they exhibit high cultural competence (in the case of a single cultural consensus model). The interview guide is designed to understand the extent to which cultural consensus models differ qualitatively. Additionally, the qualitative interviews can be used to clarify and further expand on findings from the rating and free listing phases.

\section{Participants and procedures}

Phase 3 conducted qualitative interviews with key informants from phase $2(N=25$; mean age 15.8$)$ who were highly consistent with the identified single cultural consensus model to gather in-depth information regarding the identified determinants of pregnancy and STI/HIV prevention strategies. In accordance with Kvale's suggestion that a minimum (SD) sample size of 15 (5) is acceptable to achieve saturation of themes, we recruited 25 participants. ${ }^{[22]}$ Trained study staff conducted interviews in Sesotho following semi-structured interview guides. All interviews were digitally recorded, transcribed verbatim and also translated into English.

\section{Qualitative interview guide}

Again, the qualitative interview was designed to ask participants their beliefs regarding what other girls in their community think and do to prevent pregnancy and STI/HIV rather than responding with their individual beliefs or practices. Participants first responded to questions regarding where other young women receive sexual health information and which source(s) were believed to be more accurate. Next, participants provided additional information regarding methods used to prevent pregnancy and STI/HIV and factors that motivate their use of particular methods. Additional prompts gathered in-depth information regarding available reproductive health services and young women's experiences with these services. Lastly, participants provided information regarding their needs and preferences for future interventions to prevent pregnancy and STI/HIV.

\section{Phase 4: Quantitative survey \\ Overview of phase 4 approach}

Lastly, phase 4 of CCM conducts a quantitative survey to determine the extent to which cultural consensus models are associated with differences in actual behaviours for individual participants. Unlike previous study phases, the quantitative survey asks participants to report on their beliefs and behaviours, rather than those of others in their community. This phase allows one to validate whether the identified cultural consensus model(s) and corresponding cultural competence levels map onto the particular outcome.

\section{Participants and procedures}

The survey was conducted with a convenience sample of young women ( $N=300$; mean age 15.9$)$ who had not previously participated in previous study phases. The survey was conducted via the REDCap mobile app on a tablet computer.

\section{Questionnaire measures}

The questionnaire included the phase 2 rating survey but, unlike phase 2, participants rated items for their individual beliefs. The primary outcomes of interest were participants' self-reported pregnancy prevention method use (e.g. use of contraceptive patch, pills, condoms, etc.) and STI/HIV prevention method use (e.g. condom use, STI/HIV testing, etc.). Questionnaires also assessed the following domains: $(i)$ demographics; (ii) sexual behaviours; (iii) partner communication self-efficacy and frequency; (iv) sources of sexual health information; (v) pregnancy history; (vi) HIV testing history and HIV serostatus; (vii) STI testing history and history of STIs; (viii) peer norms; (ix) parental communication; $(x)$ substance use; $(x i)$ depressive symptoms; and (xii) family beliefs.

\section{Results}

For each of the study phases, we outline the data analytic approach, utilising exemplars from our study.

\section{Phase 1}

Free listing responses are condensed to a single master list with frequencies generated for identified individual free listing responses. Depending on the number of unique items generated, one can limit the number of items selected (e.g. 20 items most frequently endorsed) or those endorsed by multiple respondents (e.g. at least 3 participants). In our study, master lists were created for each of the 4 prompts (i.e. pregnancy and STI/HIV prevention methods and factors associated with their use).

\section{Phase 2}

The analysis for phase 2 is designed to examine the extent to which factors identified in phase 1 are valued by participants and evaluate whether distinct cultural models (CMs) exist for different subpopulations. To identify CMs, cultural consensus analysis on the ratings data using UCINET software is conducted. ${ }^{[23]}$ Cultural consensus analysis is very similar to a factor analysis, but is conducted on respondents rather than items. First, eigenvalues and competence scores are generated to identify whether there is more than one CM present. Eigenvalues $\geq 3$ suggest that one $\mathrm{CM}$ is probably present, with eigenvalues $<3$ suggestive of more than one $\mathrm{CM}$ or, alternatively, that there is no single shared model. Next, by looking at those individuals who score high on the first v. second factor (or third), we can examine whether demographic characteristics (e.g., age) are associated with CMs utilising ANOVA (for continuous variables) and chi-square analyses (for dichotomous outcomes).

\section{Phase 3}

Qualitative analyses utilising key informant data from either the single CM or each of the CMs can be conducted via a variety of qualitative analytic approaches (for an overview see Ref. 24). For example, as we did in our study, one could employ a grounded theoretical approach for the qualitative analyses. ${ }^{[24,25]}$ Analyses would include the identification of key themes in the data, segmenting text, and applying codes representing each theme to each text segment; writing annotations about the data content and patterns that emerged over the course of data analyses; and engaging in a systematic retrieval and review of data by theme to characterise themes and relationships between themes. The initial classification system was then used to code a randomly selected transcript with refinement of the initial coding classification scheme based on coding discrepancies and discussion of potential coding structure revisions using a standard iterative process. ${ }^{[26,27]}$ Two independent raters then code all of the interview transcripts using the finalised codebook with discrepancies resolved by a third coder. If multiple CMs exist in the data, qualitative analyses can also elucidate the extent to which responses are consistent or differ across CMs.

\section{Phase 4}

For phase 4, one first examines the extent to which a participant's responses are consonant with each of the $\mathrm{CM}(\mathrm{s})$ previously established in phase 2. Factors identified in phase 2 are weighted according to the value established by the cultural consensus analysis. A participant's consonance with a $\mathrm{CM}$ will be equivalent to the total weighted value of each factor (e.g. identified factors influencing pregnancy prevention method selection) that they endorse. Bivariate associations employing logistic regression (for dichotomous outcomes) or linear regression (for continuous outcomes) analyses between consonance with CMs, and demographic characteristics and the outcome(s) of interest (e.g. contraceptive use history) are then examined. For each CM, one then conducts logistic regression (for dichotomous outcomes) or linear 
regression (for continuous outcomes) analyses to examine predictors of the outcome(s) of interest (e.g. condom use).

\section{Discussion and conclusion}

$\mathrm{CCM}$ is a rigorous approach that yields rich qualitative and quantitative data regarding culturally-relevant factors associated with a given health behaviour of interest. CCM utilises an iterative methodology such that initial study phases inform subsequent measure development with the final phase. The final study phase serves as a validation to examine the extent to which identified cultural consensus model(s) are associated with individual beliefs and behaviours among the population of interest. Thus, CCM assumes a 'culturally naïve' perspective with individuals' shared cultural knowledge serving as the basis for further investigation. CCM has potential utility as a methodology for examining cultural factors associated with a range of adolescent health behaviours. Data from this approach may be particularly useful to inform intervention efforts that are tailored to a unique cultural context.

The aim of the present paper was to provide an overview of CCM methods and associated data analysis approaches. We illustrated the application of CCM with our study, examining cultural factors that influence contraceptive practices and STI/HIV prevention method use among Sesotho-speaking South African adolescent girls. Despite long-standing efforts to prevent teenage pregnancy and HIV, South African adolescent girls continue to experience health disparities in the prevalence of both unintended pregnancies and HIV. Previous studies examining correlates of contraceptive and STI/HIV prevention strategies have typically focused on individuals' knowledge, attitudes, and practices. ${ }^{[10-12]}$ While this approach provides insight into individual determinants of behaviour, researchers make a priori decisions on the specific constructs to be examined and assume that knowledge, attitudes and practices are uniform across cultures. By utilising a culturally sensitive methodology, data generated from our study may facilitate a more culturally informed understanding of the reproductive health practices of South African adolescent girls.

CCM applies an ethnographic approach to understand the broader cultural context of a given behaviour. CCM has many strengths of qualitative research methods as well as quantitative statistical approaches to collect rich mixed methods. However, limitations associated with individual methodologies (e.g. self-report biases when completing a quantitative phase 4 survey) are present within CCM. There are also challenges associated with this approach. First, the multiple data collection phases are more time- and staffintensive to conduct. Further, as study phases build on previous data collection, additional time is required to optimally design subsequent data collection tools (i.e. rating survey, qualitative interview guide, quantitative survey). Thus, for some resource-constrained research contexts, CCM may not be feasible.

Despite the strengths of CCM, it has not been widely applied in public health. When considering intervention development or adaptation for a particular population, CCM offers great potential to optimally inform core content and cultural tailoring. For adolescent health, CCM may be particularly useful, given the heightened importance placed on peer norms. As illustrated by the present study, CCM expands our cultural understanding of adolescents' reproductive health behaviours and also identifies key knowledge gaps.

Acknowledgements. The support of the DST-NRF Centre of Excellence in Human Development towards this research is hereby acknowledged. Opinions expressed and conclusions arrived at, are those of the author and are not necessarily to be attributed to the CoE in Human Development. The authors gratefully acknowledge the support of the Demography and Population Studies Programme, Schools of Public Health and Social Sciences, Faculties of Health Sciences and Humanities, University of the Witwatersrand, Johannesburg, South Africa.
Author contributions. JLB served as the Principal Investigator, secured funding for the study, and provided study oversight. LM was the Site Principal Investigator and provided study oversight. JLB, JMS, CS and LM created the study's protocol. JLB drafted the manuscript and all co-authors provided critical review and feedback on the manuscript.

Funding. This study was funded by the Bill \& Melinda Gates Foundation (OPP1161907; Brown: PI). The project described was also supported by the National Center for Advancing Translational Sciences of the National Institutes of Health, under Award Number UL1TR000077. The content is solely the responsibility of the authors and does not necessarily represent the official views of the NIH or the Bill \& Melinda Gates Foundation.

Conflicts of interest. None.

1. United Nations (UN). United Nations Sustainable Development Goals. New York: UN. http://www.undp.org/content/undp/en/home/sustainable-developmentgoals.html (accessed 1 March 2018)

2. International Center for Research on Women. Sexual and reproductive health and rights. https://www.icrw.org/ (accessed 15 July 2016).

3. Dellar RC, Dlamini S, Abdool KQ. Adolescent girls and young women: Key populations for HIV epidemic control. J Intl AIDS Soc 2015;18(2):64-70.

4. McHunu G, Peltzer K, Tutshana B, Seutlwadi L. Adolescent pregnancy and associated factors in South African youth. Afr Health Sci 2012;12(4):426-434.

5. Simbayi L, Shisana O, Rehle T. South African National HIV Prevalence, Incidence and Behaviour survey, 2012. Pretoria: Human Sciences Research Council, 2014.

6. Wood K, Jewkes R. Blood blockages and scolding nurses: Barriers to adolescent contraceptive use in South Africa. Reproduct Health Matters 2006;14(27):109-118.

7. Breheny M, Stephens C. Individual responsibility and social constraint: The construction of teenage motherhood in social scientific research. Culture Health Sexuality 2009;9:333-346.

8. Panday S, Makiwane M, Ranchod C, Letsoalo T. Teenage Pregnancy in South Africa - with a Specific Focus on School-going Learners. Pretoria: Human Sciences Research Council; 2009.

9. South African Department of Health. National Contraception Service Delivery Guidelines: Within a reproductive health framework. Pretoria: Department of Health; 2003.

10. MacPhail C, Campbell C. 'I think condoms are good but, aai, I hate those things': Condom use among adolescents and young people in a southern African township. Soc Sci Med 2001;52(11):1613-1627.

11. MacPhail C, Pettifor A, Pascoe S, Rees H. Predictors of dual method use for pregnancy and HIV prevention among adolescent South African women. Contraception 2007;75(5):383-389.

12. MacPhail C, Pettifor AE, Pascoe S, Rees HV. Contraception use and pregnancy among 15-24 year old South African women: A nationally representative crosssectional survey. BMC Med 2007;5:31-39.

13. Varga CA. How gender roles influence sexual and reproductive health among South African adolescents. Stud Fam Plann 2003;34(3):160-172.

14. Williamson LM, Parkes A, Wight D, Petticrew M, Hart GJ. Limits to modern contraceptive use among young women in developing countries: A systematic review of qualitative research. Reprod Health 2009;6:3-15.

15. Bull SS, Shlay JC. Promoting 'dual protection' from pregnancy and sexually transmitted disease: A social ecological approach. Health Promotion Practice 2005;6(1):72-80.

16. Weller SC. Cultural consensus theory: Applications and frequently asked questions. Field Methods 2007;19(4):339-368.

17. Fielding-Miller R, Dunkle KL, Cooper HLF, Windle M, Hadley C. Cultural consensus modeling to measure transactional sex in Swaziland: Scale building and validation. Soc Sci Med 2016;148:25-33.

18. Dressler WW, Borges CD, Balierio MC, dos Santos JE. Measuring cultural consonance: Examples with special reference to measurement theory in anthropology. Field Methods 2005;17(4):331-355

19. Statistics South Africa. Census 2011 questionnaire 2011. http://www.statssa.gov za/?page_id=3852 (accessed 1 December 2016).

20. Massyn N, Peer N, Padarath A, Barron P, Day C. District Health Barometer 2014/15. Westville, South Africa: Health Systems Trust; 2015

21. Harris PA, Taylor R, Thielke R, Payne J, Gonzalez N, Conde JG. Research electronic data capture (REDCap) - A metadata-driven methodology and workflow process for providing translational research informatics support. J Biomed Inform 2009;42(2):377-381.

22. Kvale S. Doing Interviews. Thousand Oaks: Sage Publications, 2008

23. Borgatti SP, Everett MG, Freeman LC. Ucinet for windows: Software for social network analysis. Harvard, MA: Analytic Technologies, 2002.

24. Strauss A. Qualitative Analysis for Social Scientist. 2nd ed. London: Sage; 1987.

25. Strauss A, Corbin J. Basics of Qualitative Research. 2nd ed. London: Sage; 1998.

26. MacQueen KM, McLellan E, Kay K, Milstein B. Codebook development for team-based qualitative analysis. Cult Anthro Methods 1998;10(2):31-36.

27. Taylor SJ, Bogdan R. Working with Data: Data Analysis in Qualitative Research. Introduction to qualitative Research Methods. New York: Wiley; 1998:134-163.

Accepted 4 June 2018 